\title{
Romanian Guidelines for Nonpharmacological Therapy of IBS
}

\author{
Dan L. Dumitrascu ${ }^{1,2}$, Adriana Baban ${ }^{3,4}$, Ion Bancila ${ }^{5}$, Oana Barboi ${ }^{6,7}$, Simona Bataga ${ }^{8}$, Alexandra Chira ${ }^{1,2}$, Ioan \\ Chirila $^{7,9}$, Cristina Cijevschi Prelipcean ${ }^{6,7}$, Lidia Ciobanu ${ }^{1,10}$, Anamaria Cozma-Petrut ${ }^{1,11}$, Liliana David ${ }^{1,12}$, Mircea \\ Diculescu $^{5,13}$, Daniela Dobru ${ }^{8}$, Anca Dimitriu ${ }^{5,13}$, Eugen Dumitru ${ }^{14,15}$, Mihaela Fadgyas-Stanculete ${ }^{1,16}$, Cristian \\ Gheorghe $^{5,13}$, Georgiana-Emmanuela Gilca-Blanariü ${ }^{7}$, Adrian Goldis ${ }^{18}$, Simona Grad ${ }^{1,2}$, Melania Macarie ${ }^{8}$, Cristina \\ Marica-Sabo $^{1,2}$, Doina Miere ${ }^{1,11}$, Laurentiu Nedelcu ${ }^{19}$, Anca Negovan ${ }^{8}$, Cristina Pojoga ${ }^{4,10,20}$, Andrei-Vasile Pop ${ }^{1,2}$, Stefan- \\ Lucian Popa ${ }^{1,2}$, Paul Juergen Porr ${ }^{21}$, Flaviu Rusu ${ }^{1,2}$, Andrada Seicean ${ }^{1,10}$, Ioan Sporea ${ }^{18}$, Tudor Stroie ${ }^{5,13}$, Teodora Surdea- \\ Blaga $^{1,2}$, Cristina Tocia ${ }^{15}$, Anca Trifan ${ }^{6,7}$, Ciprian Brisc ${ }^{22}$, Vasile Drug ${ }^{6,7}$
}

See Authors affiliations at the end of the paper.

Address for correspondence: Assoc. Prof. Ciprian Brisc Faculty of Medicine and Pharmacy,

University of Oradea Oradea, Romania briscciprian@uoradea.ro
Received: 19.03.2021 Accepted: 01.04.2021

${ }^{\star}$ RSNG: Romanian Society of Neurogastroenterology

\section{ABSTRACT}

Background \& Aims: The nonpharmacological therapy in irritable bowel syndrome (IBS) is expanding rapidly. Practitioners and medical educators need to be aware of progress and changes in knowledge of this topic. The Romanian Society of Neurogastroenterology aimed to create guidelines based on best evidence on the use of nonpharmacological therapy in IBS.

Methods: A group of experts was constituted. This was divided in eleven subgroups dedicated to eleven categories of nonpharmacological therapy. The subgroups searched the literature and formulated statements and recommendations. These were submitted to vote in order to obtain consensus.

Results: The outcome of this activity is represented by the guidelines of the Romanian Society of Neurogastroenterology, presented in this paper. The recommendations are seen as complementary to the pharmacological therapy and are not intended to recommend avoiding pharmacological drugs.

Conclusions: These guidelines were elaborated by a Delphi process and represent a useful tool for physicians managing patients with IBS.

Key words: acupuncture - alternative and complementary therapy - diet - FODMAP - irritable bowel syndrome - physical activity - probiotics.

Abbreviations: BAT: body awareness therapy; CBT: cognitive behavioral therapy; GFD: gluten free diet; FMT: fecal material transplantation; FODMAP: fermentable oligo, di, monosaccharides, and polyols; IBS: irritable bowel syndrome; IBS-C: IBS with constipation; IBS-D: IBS with diarrhea; IBS-M: mixed type IBS; IBS-SSS: IBS severity scoring system; LFD: low FODMAP diet; mNICE: modified NICE diet (small frequent meals, avoid trigger foods, and avoid excess alcohol and caffeine); PMO: peppermint oil; QoL: quality of life; RCT: randomized control trial; SCFA: short-chain fatty acids; TCM: traditional Chinese medicine.

\section{INTRODUCTION}

Irritable Bowel Disease (IBS) is a chronic condition, frequently present in many gastroenterological patients. Management of these patients may be difficult and there is no single pharmacological and/or non-pharmacological treatment applicable to all patients.

The Romanian Society of Neurogastroenterology decided to develop own specific guidelines for non-pharmacological therapy of IBS due to the worldwide expansion of these therapies which are present also now in Romania. Large differences seem to exist between offered non-pharmacological therapies in different countries depending on culture, tradition and availability. We felt that our practitioners should benefit from an evidence-based document to indicate what is useful or not in the therapy of IBS. Of course, we are not overlooking the pharmacological therapy, but the focus of these guidelines for the medical practitioner is limited to the non-pharmacological alternatives, a topic that has been rarely a subject of a specific guideline.

\section{METHODS}

The Romanian Society of Neurogastroenterology identified a group of experts in the fields of IBS and nutrition. The group included gastroenterologists, general practitioners, internists, and pharmacists. From this expert group, 11 subgroups were 
created, according to the previous interests of participants, in order to propose statements and recommendations on different aspects of the topic. Using a Delphi-type approach, statements and recommendations were proposed, based on evidencebased data, obtained from a comprehensive literature search. However, included in this manuscript had to be limited to an acceptable length of the manuscript.

The statements and recommendations put to the vote in order to obtain consensus on the degree of evidence and level of recommendation. All were submitted to a vote by the participants for consensus. All the group membersvoted for all the subjects, and consensus was obtained when $>80 \%$ of respondents agreed with the items. We used the GRADE system scoring to assess the strength of evidence (Table I).

Table I. Grading of the degree of evidence

\begin{tabular}{lcl}
\hline $\begin{array}{l}\text { Degree of } \\
\text { evidence }\end{array}$ & $\begin{array}{c}\text { Identifi- } \\
\text { cation }\end{array}$ & Meaning \\
\hline $\begin{array}{l}\text { High } \\
\text { Moderate }\end{array}$ & A & $\begin{array}{l}\text { High-quality studies available; very trustworthy. } \\
\text { Several studies available, at least one of high } \\
\text { quality, others with limitations; trustworthy. } \\
\text { Low }\end{array}$ C \\
Very low & D & $\begin{array}{l}\text { limitations available; not very trustworthy. } \\
\text { No evidence available, only expert opinion; use } \\
\text { with caution. }\end{array}$ \\
\hline
\end{tabular}

The strength of the recommendation was considered strong or weak according also to GRADE. For voting, each statement was presented together with the summary of available evidence. The entire panel indicated the degree of agreement for the statements and recommendations using a 6-point Likert scale from: agree strongly $(A+)$, to disagree strongly $(\mathrm{D}+)$, with intermediate points A, A-, D-, D. Connection between contributors was kept via email.

\section{LOW FODMAP DIET}

Frequently, IBS patients may report that some foods exacerbate symptoms and therefore several of them attempt use exclusion diets. Some of these foods (cereals, fruits, vegetables, and dairy products) contain fermentable oligo, di, monosaccharides, and polyols (FODMAPs), related with pain occurrence in IBS [1]. FODMAPs are poorly absorbed in the intestine and have an osmotic action. They also represent a substrate for bacterial fermentation, resulting in the accumulation of gas and water in the intestinal lumen and colonic distension. These changes generate symptoms in patients with visceral hypersensitivity, including IBS patients [2]. Therefore, a low FODMAP diet (LFD) is expected to improve IBS symptoms [3].

A low FODMAP diet implies $<3$ g/day oligosaccharides, and $<1 \mathrm{~g} /$ day polyols [4] and consists of three phases: the elimination phase ( 4 to 6 weeks), the reintroduction phase (612 weeks), and the personalized phase. First foods containing FODMAPs are eliminated from the daily diet. If symptoms fail to improve, patients should stop the diet and other alternatives should be offered. Afterwards, FODMAPs are gradually reintroduced in the diet to identify which foods determine symptoms' recurrence, therefore allowing a personalized LFD, that can be followed for a longer period $[5,6]$. A dietitian should follow the patients through all these phases.

Gut hormones play an important role in IBS pathogenesis. Patients with IBS have a low density of gut endocrine cells, most probably related with diet and microbiota. By-products resulting from bacterial fermentation of nutrients determine a low differentiation of gut stem cells toward endocrine cells, and secondary a low secretion of gut hormones. These hormones are part of the gut-brain axis and contribute to gut motility, visceral sensitivity, and secretion. Hence, a decreased density of endocrine cells will generate gut dysmotility, visceral hypersensitivity, abnormal secretion, and finally gastrointestinal symptoms [7]. Following an LFD, the density of colonic endocrine cells tends to normalize, in parallel with symptoms improvement [8].

Statement 1.1. Low FODMAP diet improves overall IBS symptoms in majority of patients with IBS. (Quality of evidence: $C$; agreement 97.2\%.)

Statement 1.2. Low FODMAP diet is more efficient, compared to traditional dietary advice, on abdominal pain, diarrhoea, bloating and urgency symptoms. (Quality of evidence: C; agreement: 100\%).

\section{Short-term effects of a low FODMAP diet}

Several randomized controlled trials (RCT) evaluated the efficacy of the elimination phase of LFD in IBS patients. A low FODMAP diet was used for 3 to 6 weeks, and was compared to habitual diet [9], traditional dietary advice [10, 11], moderate FODMAP diet [4], modified NICE guideline recommended diet [12], high FODMAP diet [1], sham diet [13] or placebo [3]. Most studies included small numbers of patients, and given the abundance of available information on FODMAPs, the blinding was difficult to achieve. In addition, 5 metaanalyses confirmed the efficacy of LFD on IBS symptoms [5], especially on abdominal pain and bloating, but the evidence was appreciated as of low quality.

A low FODMAP diet was compared with a usual diet in a randomized control trial (RCT) which included IBS patients with bloating or diarrhea as main symptoms. Adequate symptom control was observed in $68 \%$ of patients on LFD compared to $23 \%$ on usual diet $(\mathrm{p}=0.005)$. Bloating, abdominal pain, urgency and borborygmi improved after LFD. However, stool consistency and frequency were not influenced [9]. In a crossover trial LFD was compared witha a typical Australian diet. During the LFD the overall gastrointestinal symptom score (assessed using a visual analogue scale) was lower compared with the score during typical diet (22.8 mm vs. 44.9 $\mathrm{mm}, \mathrm{p}<0.001$ ). Bloating, pain, flatulence, stool consistency also improved, and in IBS with diarrhea (IBS-D) fecal frequency significantly decreased [4]. Another RCT compared LFD with sham diet, with or without probiotics and concluded that LFD determined an adequate relief of symptoms and significantly reduced symptoms score compared with placebo [13].

Traditional dietary advice is the first-line recommendation of the British Dietetic Association in IBS [14], and consists of regular meals, chewing foods properly, avoiding large meals, caffeine, alcohol, fatty foods, spices, soft drinks, insoluble fibers, beans, cabbage, and onions. In other countries, a modified 
NICE diet (mNICE: small frequent meals, avoid trigger foods, and avoid excess alcohol and caffeine) is firstly recommended [7]. Following traditional dietary advice or mNICE diet, some FODMAPs are clearly excluded. Some studies that compared LFD with traditional dietary advice $[10-12,15,16]$, reported a similar efficacy between these dietary interventions $[10,12,16]$. Other studies reported a greater improvement of symptoms following LFD $[11,15]$. Low FODMAPs and mNICE diets showed similar efficacy on global IBS with diarrhoea (IBS-D) symptoms. However, LFD improved abdominal pain in 51\% of patients, compared to $23 \%$ ( $\mathrm{p}=0.008)$ in mNICE diet group, and determined a greater reduction in average daily scores of abdominal pain, bloating, consistency, frequency, and urgency [12]. A balanced Mediterranean diet (characterized by distribution of calories and FODMAP intake over the $24 \mathrm{~h}$ period to prevent excessive FODMAP intake at once) was not inferior to LFD and was preferred by patients [16].

Statement 1.3. In IBS patients who respond to a low FODMAP diet elimination phase, a personalized low FODMAP diet in the following months can have a favorable effect on symptoms severity (abdominal pain and distention). (Quality of evidence: D; agreement: 100\%)

\section{Long-term effects of a low FODMAP diet}

Studies reported that at long-term follow-up (> 6 months) $50-70 \%$ of patients on LFD had a satisfactory relief of symptoms [17-21]. Patients reintroduced one FODMAP subgroup per week and followed reappearance of symptoms in order to identify triggering foods, that should be avoided. Based on personal tolerance, an "adapted"/ "personalized" diet was followed. At 3-month follow-up pain frequency and severity decreased, abdominal distention diminished, IBS severity scoring system (IBS-SSS) showed mild disease in most cases and also IBS quality of life (QoL) increased. The favorable effects on symptoms and on IBS-QoL were maintained at 6-month follow-up [18]. Triggering foods for IBS symptomatology were hazelnuts and chocolate [19], fructans and free fructose containing foods [22]. In a retrospective analyses, wheat, dairy products and onions were avoided by patients following long-term modified LFD [21]. However, following a LFD for a longer time was more expensive than a habitual diet and also may impair social relationships influencing eating out with family/friends [22].

Statement 1.4. A personalized Low FODMAP diet administered for long periods of time is safe and does not produce significant changes in nutritional composition (decrease in calories, fibers and micronutrients). (Quality of evidence: D; agreement: 77.7\%)

This statement was accepted even if the agreement was $77.7 \%$ instead of $80 \%$, without a second voting round. According to this, we formulated following recommendations:

Recommendation 1.1. A low FODMAP diet may be offered to IBS patients to improve abdominal pain, bloating and/or diarrhea, for a minimum of 4 weeks (elimination phase). If no symptom improvement occurs within 4 weeks, the diet should be stopped. (Quality of evidence: C, strength of recommendation: weak; agreement: 100\%)
Recommendation 1.2. In IBS patients where symptoms improved during the elimination phase, we recommend gradual reintroduction of FODMAPs (during the next 3 months) to identify triggers, followed by a personalized diet. (Quality of evidence: $C$, strength of recommendation: weak; agreement: 100\%.)

Recommendation 1.3. A probiotic may be added to Low FODMAP diet. (Quality of evidence: C; strength of recommendation: weak; agreement: $97.2 \%$ )

\section{Low FODMAPs diet and the effects on health}

FODMAPs may have favorable effects on colonic health through the prebiotic action of oligosaccharides and the antiinflammatory properties of short-chain fatty acids (SCFAs) [23]. However, there may be some concerns regarding the longterm effects of LFD on colonic health secondary to microbiota and by-products changes.

After the elimination phase of an LFD Staudacher et al. [9] reported the decrease of the concentration and proportion of Bifidobacteria, a butyrate producer with immunomodulatory effects, which is inversely related with IBS symptoms. Another study reported a decrease of Actinobacteria, Bifidobacterium, and Faecalibacterium prausnitzii. In addition, fecal SCFAs and n-butyric acid decreased [3]. However, one trial showed that the concomitant administration of the probiotic VSL\#3 to LFD increased the abundance of Bifidobacterium species [13]. If further studies confirm this finding, the issue of microbiota alteration might be overcome by probiotic supplementation. Other concerns are related to the risk of insufficient energy and fiber intake, as reported by some RCTs [10-12]. Among micronutrients, calcium intake was significantly lower on LFD compared with a habitual diet [9]. However, in the reintroduction and later in the personalized phases, the majority of FODMAPs are reintroduced and the energy and fiber intake increased to pre-diet levels $[18,22]$.

\section{DIETARY FIBERS}

There is a long history of using fibers, starting from the Ancient Greece where bran was used for constipation up to the present days where fibers are modelling intestinal microbiota. The positive effects of dietary fibers are numerous and act in different diseases. They could decrease the risk and mortality of cardiovascular diseases, obesity, colonic diseases diabetes mellitus and they may reduce the risk of cancer correlated with age, etc.

As defined by AACC, dietary fibers are the edible parts of plants or their extracts, or analogous carbohydrates that are resistant to digestion and absorption in the human small intestine and undergo complete or partial fermentation in the large intestine [24]. Another simplified way to define dietary fibers: only dietary components that reach the colon without being absorbed in a healthy human gut [25]. Dietary fiber classification is presented in Table II [26].

Other classifications take into account not only the water solubility but also the fermentability, viscosity and gel formation. From this point of view, dietary fibers can be divided in insoluble and poorly fermented (wheat bran), soluble non viscous, readily fermented (inulina) and soluble viscous gel forming non fermented (psyllium) [28]. 
Table II. Classification of dietary fibers

1. Water insoluble (cellulose, hemicelluloses, lignins)

- Celluloses: found in all plant cell walls (wheat bran, peels of apples, pears) without effect on gastric empty or glucose absorption

- Hemicelluloses: insoluble in water but soluble in alkaline medium; are found in whole grain and decrease colonic transit time

- Lignins: non-carbohydrate polymers of aromatic alcohols are found in cereal grains, potatoes and increase stool bulk and frequency of stool

2. Water soluble (pectins, gums, muccilages, beta-glucan)

- Pectins: mixture of colloidal polysaccharides, found in bananas, apples and orange and delays gastric emptying

- Gums: found in oatmeal, legumes (GUAR, locust bean), improving the glucose metabolism and without effects on gastrointestinal function. Are frequent used as food additives; they form viscous solutions preventing aggregation of the small particles of the dispersed phase

- Muccilages: are polysaccharides from plant cells (psyllium, seeds, sea weed) and prevents desiccation of the seed endosperm. Normalizes colonic transit.

- Beta-glucans: found in barley mushrooms, yeasts, grains, oats, vitamins, minerals etc. Increases stool bulk and frequency of bowel movement.

- Soluble fibers (psyllium): are easy fermentable by gut bacteria increasing butyrate which gives energy for colonic mucosa and acts as an anti-inflammatory agent, changing the composition of microbiome, resulting in the improvement of symptoms in IBS [27].

The readily fermented fibers can lead to rapid gas formation. Poorly fermented and non-fermented fibers determine less flatulence.

Statement 2.1. Some dietary fibers may act as prebiotics. (Quality of evidence: A, agreement: $100 \%$.)

Dietary fibers may can act as prebiotic agents and influence the composition of the intestinal microbiome, altering the proportions of bacterial species and promoting the development of beneficial bacteria such as Lactobacilli and Bifidobacteria.

Fermentation of dietary fibers increases the levels of butyrate, which has anti-inflammatory properties, lowering the colonic inflammation by inducing T-cell apoptosis and suppressing interferon $-\gamma$ mediated inflammation [29].

Inulin is the most studied soluble non viscous fiber acting as a prebiotic. It is usually associated with probiotics in synbiotic supplements

The attempts to modulate the gut microbiome using prebiotics, probiotics and synbiotics are based on observations that IBS may develop after an enteral infection, the small intestinal bacterial overgrowth may have symptoms similar to those of IBS and that the colonic microbiome may be altered in patients with IBS. The use of prebiotics results in specific changes in the composition and activity of bacterial populations in the microbiome. Synbiotics, which are a mixture of prebiotics and probiotics, may have a synergistic action that promotes the development of beneficial bacterial species [30].

Statement 2.2. The quantity of fibers used in IBS vary widely based on the type (characteristics) of fibers, manufacturer and if they are associated with dietary supplements or medication. (Quality of evidence: B, agreement: $97.2 \%$.)
The American Academy of Nutrition and Dietetics, recommends $25 \mathrm{~g}$ of fiber per day for women and $38 \mathrm{~g}$ of fibers per day for men, whether they have IBS or not. Fibers are administrated to normalize the stool, to reduce abdominal pain, bloating and finally to improve the global symptoms of IBS. Higher intakes of dietary fibers can be associated with the aggravation of symptoms in IBS.

There are not enough data to answer how safe is long duration use of high quantity fibers and if they can be used without adverse effects. However, fibers seem to be good in the long term for elderly, well tolerated and without side effects compared to pharmaceutical drugs (suppositories, enemas etc) [31].

Moreover, in the adult population, the use of dietary fibers decreases the risk of cardiovascular diseases, colonic diseases and different types of cancer and normalize glycaemia and reduces LDL-cholesterol.

Statement 2.3. The quantity of fibers used in IBS vary widely based on the type (characteristics) of fibers, manufacturer and if they are associated with dietary supplements or medication. (Quality of evidence: $B$, agreement: $97.2 \%$.)

The role of fibers in IBS is still a subject of debate, with many questions which still remain unanswered. Fibers are recommended not only for the improvement of symptoms but also to increase the quality of life in patients with IBS. A review and meta-analysis conducted by the American College of Gastroenterology on the management of IBS, identified 15 RCT, involving 946 patients [30]. This review showed a statistically significant effect for fiber versus placebo. Insoluble fiber exacerbated pain and bloating in IBS and had no evident efficacy. On the other hand, soluble fibers can be recommended in the treatment of IBS to improve symptoms. The Canadian Association of Gastroenterology recommended psyllium for improving IBS symptoms and recommended against wheat bran supplementation based on a systematic review and metaanalysis of 15 RCT [32].

The evidence suggests that only soluble fibers (ispaghula, husk, psyllium) and not insoluble fibers (wheat bran) had favorable effects in IBS treatment. The addition of linseed relieves constipation, abdominal pain and bloating. In a randomized trial in primary care patients with IBS, psyllium administration reported a significant reduction in the severity of symptoms. In contrast, bran showed no clinical benefits and most of the patients did not tolerate it [33].

Since the majority of IBS patients report the generation or worsening of symptoms after food ingestion, there has been a great interest in the dietary and supplemental management of IBS patients.

Recommendation 2.1. Soluble fibers are recommended for overall symptom improvement in patients with mild to moderate IBS symptoms, especially in type IBS-C. (Quality of evidence: B, level of recommendation: weak; agreement: $100 \%$.)

The mechanism of action of soluble fibers also depends on other physical characteristics, such as fermentability, viscosity and binding capacity. Due to the fact that soluble and insoluble 
fibers frequently coexist and of the physiologic gut responses that do not depend only on solubility, it is difficult to examine separately the effect of the two fibers categories.

The role of fibers in the management of patients with IBS is controversial. A Cochrane systematic review that included 12 studies did not find any beneficial effect of bulking agents on abdominal pain, global assessment and symptom score [34]. On the other hand, in a review which analyzed the effect of different fibers on intestinal motility and fecal weight, all fiber types shortened transit time in patients with prolonged gut transit time. However, less fermentable fibers contributed most for increasing fecal weight [35]. In a systematic review and metaanalysis including 14 RCTs, soluble fibers have been found to be beneficial in the global symptom improvement of IBS [27].

Recommendation 2.2. Soluble fibres are well tolerated, have a low cost and therefore represent a reasonable first-line treatment in IBS. (Quality of evidence: B, level of recommendation: weak, agreement: $97.2 \%$.)

The availability, low cost and good tolerability make soluble fibers a recommended first-line therapy for IBS patients with mild to moderate symptoms, in conjunction with medical therapy, although the evidence to support the beneficial effect is moderate. In comparison to insoluble fibers, bloating, flatulence and abdominal distention seem to be less prominent for soluble fibers [32].

Statement 2.4. Insoluble fibers do not have any benefits in improving symptoms of IBS compared with placebo and cannot be recommended for the management of IBS. In some cases, these fibers can exacerbate abdominal pain and bloating. (Quality of evidence: A, agreement: $100 \%$.)

Dietary fiber supplementation remains a cornerstone of IBS management, although its optimal use is controversial [36]. Inadequate fibers consumption may contribute to constipation in patients with IBS with predominant constipation. Increasing dietary fibers intake is a traditional first-line treatment for patients with IBS, but insoluble fibers, such as bran, can exacerbate abdominal pain and bloating [37].

Fiber treatment may be beneficial in IBS patients with constipation (relative risk: 1.56 ; 95\%CI: $1.21-2.02$ ), but there was no evidence that fibers were effective in the relief of abdominal pain in IBS. Soluble and insoluble fibers, separately, had different effects on global IBS symptoms. Soluble fibers (psyllium, ispaghula, calcium polycarbophil) showed significant improvement (relative risk: 1.55; 95\%CI: 1.35-1.78), whereas insoluble fibers (corn, wheat bran), in some cases, worsened the clinical outcome, but there was no significant difference compared with placebo (relative risk: 0.89; 95\%CI: 0.72-1.11) [38].

In a recent meta-analysis, among the 12 studies that randomized insoluble fibers, nine used wheat bran, and one each used corn fibers, vegetable fibers, and cereal/fruit fibers. There was a broad range of doses administered for the fiber group, from 4.1 to $40 \mathrm{~g} /$ day. The duration of therapy ranged from 3 to 16 weeks. On the basis of the results of this metaanalysis the conclusion was that insoluble fibers do not have any beneficial effects in IBS [39].

\section{GLUTEN FREE DIET}

In respect to the common use of gluten free diet (GFD) in IBS and of the spread consumption of gluten-free food in normal, population, our literature search lead to the following conclusions:

Recommendation 3.1. No recommendation can be made regarding the gluten-free diet in patients with IBS. (Quality of evidence: C, strength of recommendation: weak; agreement: $94.4 \%$.)

The effect of GFD in IBS patients' outcome was studied in four RCTs and three prospective trials. The pain improved in $75-83 \%$ of cases at $4-6$ weeks of GFD, compared to $25-38 \%$ in placebo [40-42] especially in those with presence of antigliadin antibodies [40]. The IBS- symptoms severity score improved $>50$ points in $71 \%$ of patients, after 6 weeks of GFD [43] and in $67 \%, 34 \%$ or $49 \%$ respectively, after 3,4 or 6 months of GFD [44-46]. The reintroduction of gluten after 4 weeks (gluten wash-out period), produced the worsening of the overall symptoms in 59\% of the "gluten group" vs 33\% of the "placebo group" [42]. The pain occured within the first week after gluten reintroduction [42], as proved in another RCT, which reported $68 \%$ pain in gluten-free patients compared to $40 \%$ in the placebo group [47].

The GFD improved or normalized the stool frequency in RCT [48] and two prospective trials [40-46]. Also, bloating and stool consistency improved after GFD, especially in with HLA-DQ2/8- positive subjects [41-43] and worsened after gluten reintroduction $[42,43]$.

The QoL parameters, anxiety and depression scores, fatigue impact score, and Short Form-36 results [43] and the tiredness improved under GFD [42, 47].

One prospective study assessed the balanced diet, the FOODMAP diet and GFD, on the same number of patients. Although all diets improved the pain, bloating or quality of life, the balanced diet was preferred by $86 \%$ of patients, while the GFD in only $11 \%$ [16].

One meta-analysis, which included 11 trials (the above three prospective and six RCT, plus studies with functional disorders in pediatric population and one retrospective study), considered that gluten might contribute to the occurrence of gastrointestinal symptoms in patients with IBS, without indicating the GFD as a routine recommendation [49].

Recommendation 3.2. The assessment of HLA DQ2/8 for GFD in IBS-D is not recommended. (Quality of evidence: $C$; strength of recommendation: weak; agreement: 100\%.)

There is no influence of HLA DQ2/8 status on the response of IBS symptoms severity score to GFD in one RCT and two prospective trials $[43,44,47]$. The presence of HLA DQ2/8 had a sensitivity of only $25 \%$ and specificity of $52 \%$ from GFD 
responders in a prospective study [44]. However, in these patients the bloating decreased, and the depression score and vitality score improved, compared to HLA DQ2/8 negative patients [43]. In patients with IBS-D, diarrhea was resolved more frequently in HLA DQ2-positive patients with celiac disease-associated IgG antibodies $[46,48]$ or in IgG and IgA positive anti-gliadin patients [40] However, more data are needed for a final conclusion.

\section{LACTOSE FREE DIET}

The prevalence of both IBS and lactase deficiency in the general population is high; therefore, the number of people suffering from both conditions simultaneously or independently is significant. IBS patients often complain of lactose or milk intolerance and usually it is a self-reported intolerance. In a meta-analysis that included 9,041 IBS patients tested with hydrogen breath test, the prevalence of malabsorption was 56\% (95\%CI: 43-69\%) in South Asia, 50\% (95CI: 43\%-56\%) in Europe, and 21\% (95\%CI: 14-29\%) in USA [32]. Several studies, most of them non-RCTs, have been conducted to assess the prevalence of lactose malabsorption and to correlate the symptoms with objective findings on hydrogen breath test. Several other case-control studies showed that was no significant differences in lactose malabsorption prevalence between IBS patients and controls. Vernia et al. [50] compared the prevalence of lactose malabsorption in patients diagnosed with IBS ( 503 patients fulfilled the Rome criteria for IBS) with that in patients with self-reported milk intolerance (336 patients). The lactose absorption was assessed objectively by the hydrogen breath test. They found a high prevalence in both conditions: $66.9 \%$ of the subjects with IBS tested positive and $71.4 \%$ of the subjects with self-reported milk intolerance tested positive. They concluded that there was a significant overlap between the two conditions.

Vernia et al. [51] also conducted another case-control study analyzing the hydrogen breath test results following a load of lactose in IBS patients with self-reported milk intolerance. The control group was represented by patients diagnosed with IBS without self-reported milk intolerance. The conclusion of the study was that self-reported milk intolerance does not help in identifying lactose malabsorbers. Yang et al. [52] compared lactose absorption between IBS-D patients and healthy controls and also found that self-reported lactose intolerance was not associated with a positive hydrogen breath test. Gupta et al. [53] found that patients with IBS are more likely to report symptoms following lactose ingestion, but the level of breathed hydrogen was similar to that in healthy controls. Only one study found that patients with the IBS-D have a higher incidence of lactose intolerance. However, the study involved only 25 patients and 25 controls [54]. Varjú et al. [55] performed a meta-analysis and found that lactose intolerance, but not lactose malabsorption assessed by the hydrogen breath test, was more frequent among patients with IBS compared with healthy controls.

The objective studies performed using the hydrogen breath test have found a discrepancy between the prevalence of symptoms of lactose intolerance and positive test results. There was also little evidence to suggest that objective lactase deficiency was more common among IBS patients compared with healthy controls. After lactose ingestion, IBS patients reported more symptoms, but breath testing did not yield a significantly higher percentage of positive results.

According to the available data we have formulated the following recommendations:

Recommendation 4.1. We do not recommend routine testing with a lactose hydrogen breath test in IBS patients to exclude lactose malabsorption. (Quality of evidence: A; strength of recommendation: strong; agreement: $100 \%$.)

Recommendation 4.2. We do not recommend a routine lactosefree diet in IBS patients. (Quality of evidence: C; strength of recommendation: weak; agreement: $100 \%$.)

Several studies, all non-RCTs, have investigated the role of lactose-free diet or low lactose diet in IBS patients. All four studies included patients with IBS and positive lactose hydrogen breath tests, and assessed gastrointestinal symptom scores at baseline and after a variable period of a lactosefree diet [56-59]. All studies concluded that there was no improvement in gastrointestinal symptoms after a lactose-free diet or low lactose diet $(<9 \mathrm{~g} /$ day), except one small study of 16 patients [56]. Patients with IBS did not respond better to an exclusively low lactose diet ( $<9 \mathrm{~g} /$ day). However, the intake of lactose is restricted in all individuals who are following a low FODMAP diet.

Recommendation 4.3. We suggest starting a trial of milk-free diet rather than a lactose-free diet in IBS patients with a self-reported milk intolerance with a negative lactose hydrogen breath test. (Quality of evidence: D; strength of recommendation: weak; agreement: $94.2 \%$.)

Patients usually report "milk intolerance" rather than lactose intolerance per se. A subgroup of patients may be intolerant to other substances than lactose that are regularly found in milk (for example, the protein beta-casein) [60]. Further research is needed, and a possible way to resolve the issue could be to perform a RCT to compare the response to a lactose-free vs milk-free diet in patients with IBS and selfreported milk intolerance.

Recommendation 4.4. We do not recommend lactase enzyme supplementation in IBS patients. (Quality of evidence: D; strength of recommendation: weak; agreement: 100\%.)

A double-blind, cross-over study, performed by Lisker et al. [61], compared the response of the patients with confirmed lactose malabsorption to lactase and placebo and they found no correlation between symptom severity and treatment with lactase. Another study assessed the response of lactase-deficient patients with IBS to acidophilus milk with that of regular milk. The rationale was that acidophilus milk could supply the gut with additional bacterial flora as well as providing bacterial lactase. The study showed that lactase-deficient patients were as intolerant to acidophilus milk as to unaltered milk [62]. 
These studies showed that lactase supplementation in IBS patients with objective lactase deficiency did not improve symptoms, which follows the results of the studies conducted using hydrogen breath testing.

These studies showed that lactase deficiency is not responsible for symptoms associated with IBS, which follows the results of the studies conducted using hydrogen breath testing.

\section{PEPPERMINT OIL}

Peppermint oil (PMO) is obtained from the aerial parts of peppermint plant (Mentha x piperita L.) and contains L-menthol as main component and active ingredient [63]. It is an essential oil that exhibits several physiological effects within the gastrointestinal tract, including intestinal smooth muscle relaxation via calcium channels blockade, visceral antinociception via modulation of transient receptor potential channels, 5-hydroxytryptamine antagonism, modulation of histaminergic and cholinergic receptors, kappa opioid agonist activity, and antimicrobial and anti-inflammatory effects [64]. Therefore, PMO may improve IBS symptoms by targeting gastrointestinal motility, visceral hypersensitivity, the gut microbiota and the immune system [65].

There is evidence indicating benefits of PMO in the treatment of IBS, especially for patients with abdominal pain. A recent systematic review and meta-analysis of 12 RCTs in 835 patients with IBS showed that PMO compared to placebo significantly improved global IBS symptoms (RR of improvement 2.39, 95\%CI: 1.93-2.97, p<0.001) and abdominal pain (RR of improvement 1.78, 95\%CI: 1.43-2.20, $\mathrm{p}<0.001$ ) [66]. A later systematic review and network meta-analysis compared the efficacy of PMO, soluble fiber, antispasmodic drugs, and central neuromodulators in IBS. Peppermint oil was ranked first for efficacy, when using as endpoint the failure to improve global IBS symptoms after 4 to 12 weeks of treatment (RR 0.63, 95\%CI: 0.48-0.83). Nevertheless, some RCTs included in the study lack of methodological rigor, suggesting uncertainty on the findings [67].

With regard to the dosage of PMO for the treatment of IBS, most trials have tested the effects of 0.2-0.4 mL (187 to $500 \mathrm{mg}$ ), administered two or three times daily, for 2 to 8 weeks [68]. Indeed, the European Medicines Agency has recently approved PMO for the symptomatic relief of minor spasms of the gastrointestinal tract, flatulence and abdominal pain, especially in patients with IBS and recommends to adult patients a daily dose of $0.6-1.2 \mathrm{ml}$, divided in two or three times a day [69].

Relating to the safety of PMO, only a few adverse events have been reported during its use for IBS in short-term clinical trials. In the recent systematic review and meta-analysis conducted by Alammar et al. [66], the most common adverse event of oral PMO therapy in IBS was heartburn. However, this tended to be mild and transient and was also not significantly different in the IBS subjects using PMO versus placebo.

Gastro-esophageal reflux symptoms associated with PMO administration may be due to its effects as a relaxant of the lower esophageal sphincter [70]. These adverse events may be avoided by the enteric coating of PMO formulations, which facilitates delivery with sustained release to the lower gastrointestinal tract, and therefore prevents or reduces heartburn as well as improving PMO efficacy [68]. In fact, in a 4-week RCT [71], a triple enteric coated formulation of 90 mg PMO, taken two times a day, was reported to be effective at improving IBS symptoms in patients with mixed type IBS (IBS-M) or IBS-D $(n=72)$. At trial completion, there was a $40 \%$ decrease in the total IBS symptom score in the PMO group compared to baseline, superior to the $24.3 \%$ reduction observed with placebo $(\mathrm{p}=0.024)$. Moreover, the study showed PMO to be safe and well tolerated [71]. In contrast, in a more recent double-blind RCT on 190 patients with IBS, neither small-intestinal release PMO (182 mg/day) nor ileocolonic release PMO (182 mg/day) led to a statistically significant reduction in abdominal pain or increase in overall relief, after 8 weeks of treatment [72]. This is while it was hypothesized that the ileocolonic release formulation may ensure an increased efficacy of treatment due to a more targeted colonic antinociceptive effect. Nevertheless, the small-intestinal release $\mathrm{PMO}$ was superior to a placebo in improving secondary outcomes of abdominal pain, discomfort, and IBS symptom severity. Furthermore, there was a higher incidence of adverse effects in both PMO-treated groups compared to placebo but were all mild and transient [72].

Recommendation 5.1. We recommend peppermint oil to improve overall symptoms, as well as abdominal pain in IBS patients. (Quality of evidence: B; strength of recommendation: strong; agreement: $97.2 \%$ )

\section{HERBAL THERAPY}

There are only a few good quality published placebo controlled, randomized, double-blind clinical trials investigating the effect of various herbal therapies in the management of IBS [73]. The majority of them questioned the role of traditional Chinese medicine (TCM) or a multiherbal combination STW 5 (bitter candytuft, chamomile flower, peppermint leaves, caraway fruit, liquorice root, lemon balm leaves, celandine herbs, angelica root, and milk thistle fruit), in observational studies.

The last published systematic review regarding the use of herbal therapy to improve the symptoms in IBS evaluated 6,395 patients with IBS from 72 RCTs using the Rome criteria or Chinese National criteria for the diagnosis. The research concluded that the TCM combined with conventional Western medicine improved IBS symptoms compared with Western medicine alone RRs of 1.22 (95\%CI: 1.14-1.30) [74]. The authors underlined the low quality of the included trial, the differences among treatment duration and the lack of followup in most studies.

The multiherbal drug combination STW 5 has been questioned for IBS management with favorable results [75], but the outcomes were assessed using a non-standardized original questionnaire without possibility of evidence replication. A systematic review including 27 studies showed that the STW 5 had no clear benefit in IBS [76].

A recent published systematic review and meta-analysis of 21 studies evaluating the efficacy and safety of acupuncture 
combined with TCM in irritable bowel syndrome with diarrhea management, support that intervention might be effective and safe. Acupuncture combined with TCM might result in more favorable improvements compared with the control group (RR: 1.29; 95\%CI: 1.24-1.35; $\mathrm{p}=0.03$ ) [77]. A meta-analysis of 11 studies using data from 906 participants showed a significant improvement in overall clinical efficacy of TCM compared with cisapride and mosapride in patients with IBS with constipation $(\mathrm{OR}=4$; 95\%CI: 2.74-5.84; $\mathrm{p}<0.00001)$ [78].

A small, short duration placebo-controlled, randomized controlled study in 120 patients with mild to moderately severe IBS, using IBS-SSS showed that a combination of curcumin and fennel essential oil improved symptoms and the QoL in the patients with IBS compared with a placebo $(50.05 \pm 28.85 \%$ vs $26.12 \pm 30.62 \%, \mathrm{p}<0.001$ ) [79].

The heterogenous underlying pathophysiology of IBS is probably the main cause of the limited evidence obtained in IBS therapeutic trials with herbal compounds also with no clear chemical mechanism [80]. The evidence for herbal treatments derives from studies with compounds that are not regulated, and the amount of 'active ingredient' may vary among the different products. The lack of reliable information about the efficacy, using frequently non-standardized original questionnaire for outcomes' assessment and no international accepted criteria for diagnosis, without possibility of evidence replication, are the main limitations of the published studies in this field. The use of both single or compound preparations, the issues of herbal product quality and the use of personalized therapy in TCM performed in the Chinese population, limit further the studies' results and interpretations [78]. Despite the reported data coming from one study with good quality methodology [81] the result might not be used for clear recommendation in the Western population.

The consensus group concluded that even if some herbal therapies may have an effect on IBS symptoms and they have a good safe profile, there is insufficient evidence for any particular herbal product recommendations at least in our area.

Irritable bowel syndrome patients frequently use herbal therapy as first line therapy. However, there are limited scientific based data related to its efficiency. self-indication and self-administration.

Statement 6.1. Based on current data we do not recommend the use of herbal therapies for IBS. (Quality of evidence: D; strength of recommendation: weak; agreement: $94.4 \%$.)

\section{PROBIOTICS}

Probiotics are defined as "live microorganisms that, when administered in adequate amounts, confer a health benefit on the host" [82]. In the last decade, the use of probiotics in IBS has been intensively studied due to evidence that this functional gastrointestinal disorder might have an underlying microbial pathogenesis. There are studies showing differences in the composition of intestinal microbiota in IBS patients compared with healthy controls as well as unique microbial signatures associated with the severity of IBS symptoms [83, 84].

In IBS, probiotics may act by restoring intestinal dysbiosis, normalizing gut dysmotility, enhancing intestinal barrier function, reducing visceral hypersensitivity, downregulating low-grade mucosal inflammation and immune activation, and improving gut-brain communication [85]. Based on these mechanisms of the action of probiotics, numerous clinical trials have been conducted to assess their efficacy in the management of IBS. Interesting results are reported by a recent RCT, in which IBS patients treated with a probiotic formulation containing a mixture of spores from five Bacillus spp. $(n=30)$ showed improvements in the severity of symptoms, quality of life, and rectal sensation to the same degree as the patients treated with rifaximin followed by a low FODMAP $\operatorname{diet}(n=30)[86]$.

Furthermore, several systematic reviews and meta-analyses have indicated a beneficial effect of probiotics over placebo on IBS symptoms. The meta-analysis of 53 RCTs involving 5,545 patients, conducted by Ford et al. [87], reported that some particular combination probiotics, most of which contained different species of Lactobacillus, determined a lower incidence of persistence of IBS symptoms (RR: 0.79, 95\%CI 0.68-0.91] and reduced scores for flatulence (SMD: $0.29,95 \% \mathrm{CI}:-0.51$ to -0.07 ) but not for bloating.

A further meta-analysis of 35 RCTs in 3,452 patients, published by Niu et al. [88], showed a reduced risk of persistent IBS symptoms (RR: 0.79, 95\%CI: $0.70-0.89$ ) as well as a reduction in scores for abdominal pain (SMD: $-0.25,95 \% \mathrm{CI}$ : -0.36 to -0.14 ), bloating (SMD: $-0.15,95 \% \mathrm{CIL}-0.27$ to -0.03 ), and flatulence (SMD: $-0.20,95 \% \mathrm{CI}:-0.35$ to -0.05 ), with multi-strain probiotics containing one or both of the bacteria from the Lactobacillus and Bifidobacterium genera.

Moreover, the recent meta-analysis of 59 RCTs including 6,761 patients, conducted by Li et al. [89], found probiotics to reduce global IBS symptoms scores (SMD: -1.8 , 95\%CI: -0.30 to -0.06$)$. However, in contrast to Ford et al. [87] and Niu et al. [88], Li et al. [89] suggested that single probiotics may be more effective than combination probiotics in IBS symptom alleviation.

Despite the promising findings of the meta-analyses previously described [87-89], it remains difficult to draw a determinate conclusion on the efficacy of probiotics in IBS treatment and this point is also highlighted by the authors of these studies. There is difficulty in the interpretation of data due to evidence of publication bias and great heterogeneity between compared trials, in terms of individual strains or combinations of strains used, dose, duration of treatment, end points and outcomes reported, and statistical analyses.

Considering that the effects of probiotics are strainspecific and dose-specific [90], further studies are warranted to establish which probiotic strains are most efficient for IBS treatment and the conditions for their intake, such as formulations, dosage, and duration. Research should provide particular attention to the duration of treatment. In many trials, the duration of the probiotic intervention varies from 4 to 16 weeks. The systematic review of 11 RCTs published by Dale et al. [91] suggested that probiotics have a delayed effect in the alleviation of IBS symptoms and require an intervention period lasting at least 8 weeks. Indeed, the long-term effects of probiotic therapy in IBS are unclear and should be addressed in future studies. Likewise, many of the trials on probiotics use in IBS conducted to date include a relatively small sample 
size. Therefore, further studies that are well-designed and have a large sample size are required.

Finally, the findings regarding the safety of probiotics in IBS are limited and conflicting. In fact, it has been acknowledged that safety outcomes are inconsistently assessed and reported in probiotic intervention studies [92]. In IBS, the incidence of adverse events was not significantly greater among patients treated with probiotics than among those assigned to placebo, according to both the meta-analysis by Ford et al. [87] (RR: 1.09, 95\%CI: 0.91-1.29) and the meta-analysis by Li et al. [89] (RR: 1.07, 95\%CI: 0.92-1.24). In contrast, the meta-analysis conducted by Niu et al. [88] reported a higher incidence of any adverse event in patients who received probiotics versus those who received placebo (RR: $1.21,95 \% \mathrm{CI}: 1.02-1.44)$. These results suggest more evidence is required concerning the safety of probiotics in IBS.

Recommendation 7.1. In patients with IBS, we recommend the use of probiotics as an alternative therapy in trials of limited duration. (Quality of evidence: B; strength of recommendation: weak; agreement: 97.2\%.)

\section{PHYSICAL EXERCISE}

The data presented so far in the literature indicate that the promotion of physical activity in the general population can help prevent the occurrence of IBS [93]. Exercise is proven beneficial for health because it reduces the risk of cardiovascular disease, endocrine disorders and lowers levels of anxiety and depression [94]. Regular physical activity can help relieve constipation, promote bowel movements and improve bloating [95]. Patients experiences with the effects of physical activity on IBS symptoms are not really known. This knowledge is required to enable adequate support from health professionals. In some patient groups it is difficult to motivate patients to change their lifestyle [96]. Physical activity counteracts the effects of stress [97]. Compared to physically active people (1 hour / week), those with sedentary physical activity ( $<1$ hour / week) were 1.27 times more likely to have IBS [98]. There was a significant difference in improving the IBS-SSS score between the physical activity group and the control group. Here is a review of main physical activities and their possible effects on IBS symptoms.

Walking and cycling: a moderate increase in physical activity within 12 weeks, $20-60 \mathrm{~min} /$ day of moderate to vigorous physical activity, 3-5 days a week, may improve symptoms and can be effective against constipation.

Aerobic exercise may improve: constipation, abdominal pain, abdominal distension, depression and anxiety. A lowto-moderate intensity exercise training attenuated symptoms in sedentary IBS patients. Symptom improvement might have been associated with a reversal of the ratio of antiinflammatory/proinflammatory cytokines and blood redox homeostasis, suggesting that a low to moderate intensity exercise training program may have immune and redoxmodulating functions [99]. Thus, aerobic exercise appears to be a simple, acceptable, effective, feasible and eligible treatment approach for patients with IBS.
Swimming and running/jogging were studied; at a 12 week follow-up compared to baseline, the symptoms were significantly lower in the physical activity group. However, the IBS-SSS score was also significantly lower in those with physical activity [100]. Constipation symptoms were significantly better in the mountaineering group than in the control group in a study comparing the effects of hiking in patients with IBS [101]. Baduanjin qigong exercise is a type of traditional Chinese fitness exercise that involves the following 4 parts: mood relaxation, breathing adjustment, organ regulation and shape readjustment. The overall efficiency, symptoms and stool characteristics were significantly better in Baduanjin qigong active elderly IBS patients with constipation (IBS-C) [102]. Yoga has similar positive effects on IBS symptoms such as walking. Physiotherapy is a useful resource when discussing physical activity. Patients with depression needed the active support of a physiotherapist to overcome their own resistance in order to participate in an exercise intervention.

Body awareness therapy $\left(\mathrm{BAT}^{\mathrm{TM}}\right)$ consists of simple structured movement exercises, based on human anatomical and physiological conditions to achieve optimal movement dynamics. BAT ${ }^{\mathrm{m} m}$ exercises aim to help the body find its natural posture, thus facilitating the circulatory, muscular, nervous and respiratory systems to regain their natural function. $\mathrm{BAT}^{\mathrm{TM}}$ is used to treat various stress and pain conditions in all Nordic countries, as well as in Scotland, Switzerland, Austria, the Netherlands, Spain and Turkey [103]. Posture, breathing and muscle tension, along with the function and mobility of the internal organs are affected by body-mind training. It is assumed that body-mind therapies work through a physiological transformation achieved through the autonomic nervous system.

Recommendation 8.1. Physical exercise may be useful in IBS. Regular exercise can help manage some symptoms in IBS and can be a primary treatment in IBS-C. (Quality of evidence: $C$; strength of recommendation: low; agreement: $97.2 \%$.)

\section{ACUPUNCTURE}

A meta-analysis by Zheng et al. [105] analyzed 41 RCTs $(3,440$ subjects) for the assessment of acupuncture efficacy in IBS. The results show no significant difference between acupuncture and sham on IBS symptoms and QoL (SMD: 0.18, 95\%CI: -0.26 to $0.63, \mathrm{p}=0.42$; SMD: $-0.10,95 \% \mathrm{CI}:-0.31$ to $0.11, \mathrm{p}=0.35$ ) [105]. Further, the study demonstrated that acupuncture was more effective compared with western medicine in alleviating IBS symptomatology (RR: 1.17, $95 \% \mathrm{CI}: 1.12-1.23, \mathrm{I} 2=0 \%, \mathrm{p}<0.00001)$, and the positive effect lasted about 3 months [105]. From 8 RCTs which compared acupuncture with sham acupuncture, 3 RCT concluded show a significant benefit of acupuncture in treating abdominal pain, discomfort, and stool frequency [105]. A meta-analysis performed by Chao et al. [106] analyzed 6 RCT about the effectiveness of acupuncture in IBS [106]. The results showed that the relative risk for clinical improvement with acupuncture was 1.75 (95\%CI: 1.24-2.46, p=0.001) and the authors 
concluded that acupuncture had statistically significant positive effects in the reduction of IBS symptoms [106]. A study by Wu et al. [107] conducted an overview of systematic reviews and a network of meta-analysis in order to evaluate the comparative effectiveness of acupuncture and related therapies. A total of 2,141 IBS patients from 27 RCT were included in the study and the results demonstrated that both classic acupuncture using metallic needles and electro-acupuncture were superior in improving IBS symptomatology compared to other therapies. The authors concluded that IBS patients with no response to first-line conventional therapies or antidepressant agents may consider acupuncture as an alternative [107].

Recommendation 9.1. There is not enough evidence to recommend acupuncture as therapy of IBS. (Quality of evidence: D; strength of recommendation: low; agreement: $100 \%$.)

There is no consensus yet in respect to the role of acupuncture in IBS. Most trials were of poor quality because the results were heterogeneous and were obtained from interventions and control groups. It should also be taken into account that so far, all RCTs did not analyse the long-term effects of acupuncture on IBS evolution [105-113]. Another major limit of most RCT is represented by the fact that the level of the therapist training is not mentioned nor the acupuncture points or technique. The majority of RCTs did not mention any information about the effects of acupuncture on the IBS subtype. We also mention that most studies report that adverse events are rare and include local bleeding and local pain [114, 115]. Extremely rare, serious complications can include nerve injury and infections $[114,115]$. Finally, it cannot be ruled if acupuncture is effective in IBS, and more high-quality studies are required.

\section{PSYCHOLOGICAL THERAPIES}

Psychotherapy is frequently used in IBS, mainly in patients with severe symptoms or with important pathogenic contribution of psychosocial factors.

Statement 10.1. Psychological therapies are useful in IBS. (Quality of evidence: B; agreement: $100 \%$.)

IBS is thought to result from the interaction between biological, psychological, and social factors [116]. Early life events, psychological distress, and negative coping style may play essential roles in the pathogenesis of IBS. The use of maladaptive coping strategies positively correlates with symptom severity and degree of anxiety and depression among patients [117].

The lack of a universally effective medical treatment has led to various psychological treatments recommended in patients who do not respond to medical therapy. Psychotherapy options contain psychoeducation, cognitive-behavioral therapy (CBT), psychodynamic psychotherapy, hypnotherapy, mindfulness, relaxation therapy.

The effect of psychological interventions in IBS was studied in 41 RCTs containing 4072 adult participants, with a minimum duration of therapy of 4 weeks and a minimum duration of follow-up of 4 weeks. These RCTs compared different psychological therapies with each other or with a control intervention $[116,118]$.

The psychological interventions with the highest efficacy included cognitive-behavioral therapy with two different approaches: the self-administered and minimal contact approach (RR: $0.61 ; 95 \% \mathrm{CI}: 0.45-0.83, \mathrm{p}=0.66)$, or face-to-face approach (RR: 0.62 ; 95\%CI: $0.48-0.80$, P score 0.65 ) and also included gut-directed hypnotherapy (RR: 0.67; 95\%CI: 0.49 to $0.91, \mathrm{p}=0.57$ ) [116].

A trial showed a reduction of $50 \%$ of digestive symptoms, anxiety, and depression in the cognitive behavioral therapy (CBT) group [119]. Another RCT demonstrated a 42\% decrease in gastrointestinal symptoms in patients with IBS treated using an online CBT program compared to the control group [120].

After 15 and 18 months, the follow-up revealed the same positive results in the psychological intervention group [121]. Another study compared standard CBT with home-based CBT with minimal therapist contact and also with education and found improvement in gastrointestinal symptoms in 61\% patients from the minimal contact CBT group, $55 \%$ patients from the CBT group, and $44.8 \%$ patients from the education group [121]. At six months after the end of treatment, there was a significant difference between minimal contact CBT (58.4\%) and education (44.8\%) regarding digestive symptom improvement [122]. This trial showed similar efficacy of minimal contact CBT and standard CBT; therefore, this kind of psychotherapy could be delivered online or by telephone, most probably in a more cost-effective manner.

Gut-directed hypnotherapy (both as an individual or group approach) has been demonstrated to produce a reduction in IBS symptoms $(p<0.05)$ [123]. It seems to be superior to medication and has a long-term effect. $60.8 \%$ of patients treated with hypnotherapy improved vs. $40.9 \%$ treated with standard medical treatment with the effect lasting over 15 months $(54.3 \%$ of gut-related hypnotherapy patients and $25.0 \%$ of controls improved [124]. It also has a positive effect on the quality of life, somatic and psychological symptoms. In a RCT published in 2019, the improvement of symptoms was $40.8 \%$ in the individual hypnotherapy group, $33.2 \%$ in the group hypnotherapy group and $16.7 \%$ in the control group at three months. Hypnotherapy was more effective than control at three months $(\mathrm{p}=0.0240)$ and 12 months $(\mathrm{p}=0.0185)$. Group hypnotherapy was non-inferior to individual hypnotherapy [125].

Among trials recruiting only patients with refractory symptoms, CBT and gut-directed hypnotherapy were more efficacious than either education and/or support or routine care, and CBT via the telephone, contingency management, CBT via the internet, and dynamic psychotherapy were all superior to routine care [116].

Recommendation 10.1. Psychotherapy should be considered for people with IBS who do not respond to pharmacological treatments after 12 months and who develop a refractory IBS. (Quality of evidence: C; strength of recommendation: low; agreement: $97.2 \%$.) 
Recommendation 10.2. Psychotherapy cannot be recommended routinely in patients with IBS. It should be indicated in individual cases (with refractory symptoms and/or psychiatric comorbidities) but remains subject to the availability of appropriate resources and expertise. (Quality of evidence: B; strength of recommendation: strong; agreement: 100\%)

\section{FECAL MATERIAL TRANSPLANTATION}

The use of fecal material transplantation (FMT) has been considered for IBS, since the role of dysbiosis in this setting was highly investigated [84, 126-128]. Even though the latest published RCT identified FMT as an effective treatment for IBS, regardless of the IBS subtype, it also highlighted the importance of using a well-defined donor and adequate quantity of transplant as main prerequisites for successful FMT [129]. The results of several RCTs currently available [129-135] are conflicting and difficult to compare due to several differences among the trials: size of patient cohorts, prior patient treatment, type of donors used, amount of the transplants, route of administration. Moreover, inconsistent results from RCTs could also be due to other factors, such as the placebo effect, considering prior stated relative placebo responses for IBS symptom severity of $41.4 \%$ and for quality of life between $20 \%$ and 125\% [136]. A recent meta-analysis including all available RCTs on FMT and also single-arm trials identified crude placebo response rates in RCTs comparable to the response to FMT in single-arm trials. This finding suggests that better outcomes of FMT from single-arm trials would be mainly in the context of a placebo effect [137]. Consequently, current evidence from RCTs and meta-analyses $[137,138]$ does not provide a solid ground for an overall clinical benefit in using FMT for global IBS symptoms. It is still uncertain whether FMT is efficacious in IBS, especially considering discrepant results among RCTs in subgroup analysis. Further high-quality clinical trials are needed, including an appropriate control for FMT and also a better characterization of microbiota profile at baseline.

Recommendation 11.1. FMT should not be routinely used for IBS and should currently be limited to the research setting for this indication. (Quality of evidence: C; strength of recommendation: low; agreement: $97.2 \%$.)

\section{DISCUSSION}

Irritable bowel syndrome is characterized by chronic abdominal pain associated with changes in the frequency and consistency of stools, without organic involvement according to present Rome IV definition. A recent multicenter worldwide rigorous study gave a very low prevalence of $1.5 \%$ (1.3-1.7) for IBS using strict definition. However, prevalence for nonspecific functional bowel disorders is quite high: 16.0 (15.516.5) [130]. The pathophysiology of IBS is not fully known, the disease being considered multifactorial. This aspect makes the present treatments unsatisfactory for all patients, the management of symptoms being sometimes difficult. In addition, this condition leads to high costs, absenteeism from work and an unsatisfactory quality of life [140].

Along with drug therapy, non-pharmacological therapy can play an important role in the management of these patients and this paper attemps to to guide the medical practitioners in this regard. The LOW FODMAP diet recommends reducing or excluding fermentable foods that can influence pain in IBS, due to reduced absorption and osmotic action [3]. This diet could be tested in patients and if the exclusion does not improve symptoms, it can be stopped $[5,6]$. Although there is a low quality of evidence, there were five meta-analysis, which highlighted the effectiveness of LFD in relieving symptoms [5]. However, there are also studies linking LFD with a decrease in the concentration of Bifidobacteria, insufficient energy intake and a decrease in calcium intake, which means this diet should not be recommended for all IBS patients [3, 9-13]

Fibers have been used for long time in the diet of patients. Recent data are more subtle in the recommendations. It is recognized that insoluble fibers may cause an increase in pain intensity and excessing bloating in IBS, unlike soluble fibers, which can be recommended in the treatment of this condition $[32,33]$. Sometimes, however, it is difficult to separate soluble and insoluble fibers because they frequently coexist naturally in food components [34].

Regarding the GFD there are some prospective studies, the results of which oppose for the reduction of pain in a significant percentage, up to $83 \%$, if the diet is maintained for $4-6$ weeks and the reintroduction of gluten worsened the symptoms in a significant percentage $[40,42,44]$. However, there is some reluctance on the part of patients to accept this diet [16]; only $11 \%$ of the patients studied accepted GFD, $86 \%$ preferring a balanced diet, but there is also a meta-analysis which, although recognizing the role of gluten as a possible cause of symptoms does not indicate this regimen as a permanent recommendation [49].

Another chapter discussed in the literature is the lactose-free diet in IBS; there is generally a discrepancy between patients self-reported lactose intolerance and the positive results of confirmatory tests. Some meta-analyses have discordant results regarding the prevalence of lactose malabsorption and IBS on different continents [32]. If, however, the prevalence of lactose malabsorption was compared in patients diagnosed with IBS, to self-reported patients with milk intolerance, a high prevalence was found in both situations (66.9\% IBS and $71.4 \%$ selfreported) [50]. There are some studies that have not shown any improvement in IBS symptoms after a low-lactose or lactose-free diet [56]. It is necessary to differentiate between, self-reported milk intolerance and actual lactose intolerance, as there are other products in milk that may cause malabsorption [60).

Peppermint oil was studied in patients with IBS symptoms. A meta-analysis of 12 studies and 835 patients showed improvement in IBS symptoms and pain syndrome in the PMO group compared with placebo [66], attempting a comparison between PMO, soluble fiber, antispasmodics and central neuromodulators, but without a methodological rigor of comparative calculations [67]. The EMA also approved the use of PMOs to relieve IBS symptoms, minor spasms, flatulence and pain; no significant side effects have been reported with the use of PMO. 
Herbal therapy has limited evidence for IBS treatment efficacy, mainly due to heterogeneous pathophysiology and also due to the amount of active substances which is not standardized between different products [80].

The use of probiotics may have a beneficial effect, proven in some meta-analysis, but there are some contradictory issues related to the safety of their use, with an inconsistent assessment and safety reporting between different studies [92]. However, probiotics can restore intestinal dysbiosis, normalize intestinal dysmotility, and help improve intestinal barrier function, reduce mucosal inflammation, and reduce visceral hypersensitivity [86].

Exercise may be useful in the management of IBS [93, 94]. In addition, physical activity reduces the effects of stress [97], but no excessive physical effort is indicated, and exercise programs should be customized, depending on age and pathology.

Acupuncture can be used as an alternative in patients with IBS without a response to conventional first-line therapies, or to antidepressants [107], but there is no consensus on the role of acupuncture in IBS [105-113].

In the treatment of IBS, psychotherapy is quite often used. There are psychological methods with greater effectiveness, such as cognitive-behavioral therapy [116]. Some studies indicate an improvement in digestive symptoms as well as anxiety and depression by up to $50 \%$ [119].

Starting from the role of dysbiosis in the pathophysiology of IBS, FMT was also tested. There are contradictory studies [129-135], which are also difficult to compare, and the results are generally inconsistent, so that current evidence and metaanalysis do not provide a solid ground for the clinical benefit of FMT. [137, 138].

\section{CONCLUSIONS}

There are several non-pharmacological therapeutic alternatives for IBS. They can be used alone or associated with pharmacological therapy. As, in general, the quality of evidence and the levels of recommendation are variable, these therapeutic interventions should be individualized.

\section{Conflicts of interest: None to declare}

Authors' affiliations: 1) Iuliu Hatieganu University of Medicine and Pharmacy, Cluj-Napoca; 2) $2^{\text {nd }}$ Department of Internal Medicine; 3) Dept. of Psychology; 4) Babes-Bolyai University, Cluj-Napoca; 5) Center of Gastroenterology and Hepatology Fundeni Clinical Institute, Bucharest; 6) Institute of Gastroenterology and Hepatology Iasi; 7) Gr.T. Popa University of Medicine and Pharmacy, Iasi; 8) George E. Palade University of Medicine, Pharmacy, Sciences and Tehnology, Targu-Mures; 9) Department of Environmental Health,National Institute of Public Health-RCoPH Iasi; 10) Regional Institute of Gastroenterology and Hepatology, Cluj-Napoca; 11) Department of Bromatol, Hygiene, Nutrition; 12) Department of Nursing; 13) Carol Davila University of Medicine and Pharmacy Bucharest; 14) Research Center for the Morphological and Genetic Study in Malignant Pathology; 15) Faculty of Medicine, Ovidius University Constanța; 16) Department of Neurosciences; 17) $1^{\text {st }}$ Medical Department, Iasi; 18) Department of Gastroenterology and Hepatology Victor Babeș University of Medicine and Pharmacy, Academy of Medical Science, Research Center, Timișoara;
19) Faculty of Medicine, Transylvania University, Brasov; 20) Department of Clinical Psychology and Psychotherapy, International Institute for Advanced Study of Psychotherapy and Applied Mental Health, Cluj-Napoca; 21) Polisano MedLife Clinic, Sibiu; 22) Faculty of Medicine and Pharmacy, University of Oradea.

Authors' contribution: D.L.D. suggested the guidelines, designed the structure and methodology, identified co-authors and revised the text. VD and $\mathrm{CB}$ wrote the introductory and discussion parts. AVP checked all the references. All authors contributed to the writing of the manuscript, to the elaboration of statements and recommendations, voted them and approved the final version of the text.

\section{REFERENCES}

1. McIntosh K, Reed DE, Schneider T, et al. FODMAPs alter symptoms and the metabolome of patients with IBS: a randomised controlled trial. Gut 2017;66:1241-1251. doi:10.1136/gutjnl-2015-311339

2. Gibson PR, Halmos EP, Muir JG. Review article: FODMAPS, prebiotics and gut health-the FODMAP hypothesis revisited. Aliment Pharmacol Ther 2020;52:233-246. doi:10.1111/apt.15818

3. Hustoft TN, Hausken T, Ystad SO, et al. Effects of varying dietary content of fermentable short-chain carbohydrates on symptoms, fecal microenvironment, and cytokine profiles in patients with irritable bowel syndrome. Neurogastroenterol Motil 2017;29:e12969. doi:10.1111/ nmo.12969

4. Halmos EP, Power VA, Shepherd SJ, Gibson PR, Muir JG. A diet low in FODMAPs reduces symptoms of irritable bowel syndrome. Gastroenterology 2014;146:67-75.e5. doi:10.1053/j.gastro.2013.09.046

5. Dionne J, Ford AC, Yuan Y, et al. A Systematic Review and MetaAnalysis Evaluating the Efficacy of a Gluten-Free Diet and a Low FODMAPs Diet in Treating Symptoms of Irritable Bowel Syndrome. Am J Gastroenterol 2018;113:1290-1300. doi:10.1038/s41395-0180195-4

6. Rej A, Aziz I, Tornblom H, Sanders DS, Simrén M. The role of diet in irritable bowel syndrome: implications for dietary advice. J Intern Med 2019;286:490-502. doi:10.1111/joim. 12966

7. El-Salhy M, Hatlebakk JG, Hausken T. Diet in Irritable Bowel Syndrome (IBS): Interaction with Gut Microbiota and Gut Hormones. Nutrients. 2019;11:1824. doi:10.3390/nu11081824

8. Mazzawi T, Hausken T, Gundersen D, El-Salhy M. Dietary guidance normalizes large intestinal endocrine cell densities in patients with irritable bowel syndrome. Eur J Clin Nutr 2016;70:175-181. doi:10.1038/ ejcn.2015.191

9. Staudacher HM, Lomer MC, Anderson JL, et al. Fermentable carbohydrate restriction reduces luminal bifidobacteria and gastrointestinal symptoms in patients with irritable bowel syndrome. J Nutr 2012;142:1510-1518. doi:10.3945/jn.112.159285

10. Böhn L, Störsrud S, Liljebo T, et al. Diet low in FODMAPs reduces symptoms of irritable bowel syndrome as well as traditional dietary advice: a randomized controlled trial. Gastroenterology 2015;149:13991407.e2. doi:10.1053/j.gastro.2015.07.054

11. Zahedi MJ, Behrouz V, Azimi M. Low fermentable oligo-di-monosaccharides and polyols diet versus general dietary advice in patients with diarrhea-predominant irritable bowel syndrome: A randomized controlled trial. J Gastroenterol Hepatol 2018;33:1192-1199. doi:10.1111/jgh.14051

12. Eswaran SL, Chey WD, Han-Markey T, Ball S, Jackson K. A Randomized Controlled Trial Comparing the Low FODMAP Diet vs. Modified NICE 
Guidelines in US Adults with IBS-D. Am J Gastroenterol 2016;111:18241832. doi:10.1038/ajg.2016.434

13. Staudacher HM, Lomer MCE, Farquharson FM, et al. A Diet Low in FODMAPs Reduces Symptoms in Patients With Irritable Bowel Syndrome and A Probiotic Restores Bifidobacterium Species: A Randomized Controlled Trial. Gastroenterology 2017;153:936-947. doi:10.1053/j.gastro.2017.06.010

14. McKenzie YA, Bowyer RK, Leach H, et al. British Dietetic Association systematic review and evidence-based practice guidelines for the dietary management of irritable bowel syndrome in adults (2016 update). J Hum Nutr Diet 2016;29:549-575. doi:10.1111/jhn.12385

15. Patcharatrakul T, Juntrapirat A, Lakananurak N, Gonlachanvit S. Effect of Structural Individual Low-FODMAP Dietary Advice vs. Brief Advice on a Commonly Recommended Diet on IBS Symptoms and Intestinal Gas Production. Nutrients 2019;11:2856. doi:10.3390/nu11122856

16. Paduano D, Cingolani A, Tanda E, Usai P. Effect of Three Diets (LowFODMAP, Gluten-free and Balanced) on Irritable Bowel Syndrome Symptoms and Health-Related Quality of Life. Nutrients 2019;11:1566 doi:10.3390/nu11071566

17. Peters SL, Yao CK, Philpott H, Yelland GW, Muir JG, Gibson PR. Randomised clinical trial: the efficacy of gut-directed hypnotherapy is similar to that of the low FODMAP diet for the treatment of irritable bowel syndrome. Aliment Pharmacol Ther 2016;44:447-459. doi:10.1111/apt.13706

18. Harvie RM, Chisholm AW, Bisanz JE, et al. Long-term irritable bowel syndrome symptom control with reintroduction of selected FODMAPs. World J Gastroenterol 2017;23:4632-4643. doi:10.3748/wjg.v23.i25.4632

19. Gravina AG, Dallio M, Romeo M, et al. Adherence and Effects Derived from FODMAP Diet on Irritable Bowel Syndrome: A Real Life Evaluation of a Large Follow-Up Observation. Nutrients 2020;12:928. doi:10.3390/nu12040928

20. de Roest RH, Dobbs BR, Chapman BA, et al. The low FODMAP diet improves gastrointestinal symptoms in patients with irritable bowel syndrome: a prospective study. Int J Clin Pract 2013;67:895-903. doi:10.1111/ijcp.12128

21. Maagaard L, Ankersen DV, Végh Z, et al. Follow-up of patients with functional bowel symptoms treated with a low FODMAP diet. World J Gastroenterol 2016;22:4009-4019. doi:10.3748/wjg.v22.i15.4009

22. O'Keeffe M, Jansen C, Martin L, et al. Long-term impact of the lowFODMAP diet on gastrointestinal symptoms, dietary intake, patient acceptability, and healthcare utilization in irritable bowel syndrome. Neurogastroenterol Motil 2018;30:e13154. doi:10.1111/nmo.13154

23. Guarino MPL, Altomare A, Emerenziani S, et al. Mechanisms of Action of Prebiotics and Their Effects on Gastro-Intestinal Disorders in Adults. Nutrients 2020;12:1037. doi:10.3390/nu12041037

24. American Association of Cereal Chemists (AAC). The definition of dietary fiber. Report of the Dietary Fiber Definition Committee to the Board of Directors of the AAC 2001;46:112-126.

25. Ha MA, Jarvis MC, Mann JI. A definition for dietary fibre. Eur J Clin Nutr 2000;54:861-864. doi:10.1038/sj.ejen.1601109

26. Narayanan S, Pitchumoni CS. Dietary Fiber. In: Pitchumoni CS, Dharmarajan TS, editors. Geriatric Gastroenterology. Cham: Springer International Publishing; 2020:1-16.

27. Moayyedi P, Quigley EM, Lacy BE, et al. The effect of fiber supplementation on irritable bowel syndrome: a systematic review and meta-analysis. Am J Gastroenterol 2014;109:1367-1374. doi:10.1038/ajg.2014.195

28. Cozma-Petruţ A, Loghin F, Miere D, Dumitraşcu DL. Diet in irritable bowel syndrome: What to recommend, not what to forbid to patients! World J Gastroenterol 2017;23:3771-3783. doi:10.3748/wjg.v23.i21.3771
29. El-Salhy M, Ystad SO, Mazzawi T, Gundersen D. Dietary fiber in irritable bowel syndrome (Review). Int J Mol Med 2017;40:607-613. doi:10.3892/ ijmm.2017.3072

30. Ford AC, Moayyedi P, Chey WD, et al. American College of Gastroenterology Monograph on Management of Irritable Bowel Syndrome. Am J Gastroenterol 2018;113(Suppl 2):1-18. doi:10.1038/ s41395-018-0084-x

31. Muir J. An Overview of Fiber and Fiber Supplements for Irritable Bowel Syndrome. Gastroenterol Hepatol (N Y) 2019;15:387-389.

32. Moayyedi P, Andrews CN, MacQueen G, et al. Canadian Association of Gastroenterology Clinical Practice Guideline for the Management of Irritable Bowel Syndrome (IBS). J Can Assoc Gastroenterol 2019;2:6-29. doi:10.1093/jcag/gwy071

33. Bijkerk CJ, de Wit NJ, Muris JW, Whorwell PJ, Knottnerus JA, Hoes AW. Soluble or insoluble fibre in irritable bowel syndrome in primary care? Randomised placebo controlled trial. BMJ 2009;339:b3154. doi:10.1136/ bmj.b3154

34. Ruepert L, Quartero AO, de Wit NJ, van der Heijden GJ, Rubin G, Muris JW. Bulking agents, antispasmodics and antidepressants for the treatment of irritable bowel syndrome. Cochrane Database Syst Rev 2011;(8):Cd003460. doi:10.1002/14651858.CD003460.pub3

35. de Vries J, Birkett A, Hulshof T, Verbeke K, Gibes K. Effects of Cereal, Fruit and Vegetable Fibers on Human Fecal Weight and Transit Time: A Comprehensive Review of Intervention Trials. Nutrients 2016;8:130. doi:10.3390/nu8030130

36. Lacy BE, Mearin F, Chang L, et al. Bowel Disorders. Gastroenterology 2016;150:1393-1407.e5. doi:10.1053/j.gastro.2016.02.031

37. Ford AC, Lacy BE, Talley NJ. Irritable Bowel Syndrome. N Engl J Med 2017;376:2566-2578. doi:10.1056/NEJMra1607547

38. Bijkerk CJ, Muris JW, Knottnerus JA, Hoes AW, de Wit NJ. Systematic review: the role of different types of fibre in the treatment of irritable bowel syndrome. Aliment Pharmacol Ther 2004;19:245-251. doi:10.1111/j.0269-2813.2004.01862.x

39. Nagarajan N, Morden A, Bischof D, et al. The role of fiber supplementation in the treatment of irritable bowel syndrome: a systematic review and meta-analysis. Eur J Gastroenterol Hepatol 2015;27:1002-1010. doi:10.1097/MEG.0000000000000425

40. Pinto-Sanchez MI, Nardelli A, Borojevic R, et al. Gluten-Free Diet Reduces Symptoms, Particularly Diarrhea, in Patients With Irritable Bowel Syndrome and Antigliadin IgG. Clin Gastroenterol Hepatol 2020. doi:10.1016/j.cgh.2020.08.040

41. Shahbazkhani B, Sadeghi A, Malekzadeh R, et al. Non-Celiac Gluten Sensitivity Has Narrowed the Spectrum of Irritable Bowel Syndrome: A Double-Blind Randomized Placebo-Controlled Trial. Nutrients 2015;7:4542-4554. doi:10.3390/nu7064542

42. Zanwar VG, Pawar SV, Gambhire PA, et al. Symptomatic improvement with gluten restriction in irritable bowel syndrome: a prospective, randomized, double blinded placebo controlled trial. Intest Res 2016;14:343-350. doi:10.5217/ir.2016.14.4.343

43. Aziz I, Trott N, Briggs R, North JR, Hadjivassiliou M, Sanders DS Efficacy of a Gluten-Free Diet in Subjects With Irritable Bowel Syndrome-Diarrhea Unaware of Their HLA-DQ2/8 Genotype. Clin Gastroenterol Hepatol 2016;14:696-703.e1. doi:10.1016/j. cgh.2015.12.031

44. Barmeyer C, Schumann M, Meyer T, et al. Long-term response to gluten-free diet as evidence for non-celiac wheat sensitivity in one third of patients with diarrhea-dominant and mixed-type irritable bowel syndrome. Int J Colorectal Dis 2017;32:29-39. doi:10.1007/s00384-0162663-x 
45. Hajiani E, Masjedizadeh A, Shayesteh AA, Babazadeh S, Seyedian SS. Comparison between gluten-free regime and regime with gluten in symptoms of patients with irritable bowel syndrome (IBS). J Family Med Prim Care 2019;8:1691-1695. doi:10.4103/jfmpc.jfmpc_464_18

46. Wahnschaffe U, Schulzke JD, Zeitz M, Ullrich R. Predictors of clinical response to gluten-free diet in patients diagnosed with diarrheapredominant irritable bowel syndrome. Clin Gastroenterol Hepatol 2007;5:844-850. doi:10.1016/j.cgh.2007.03.021

47. Biesiekierski JR, Newnham ED, Irving PM, et al. Gluten causes gastrointestinal symptoms in subjects without celiac disease: a double-blind randomized placebo-controlled trial. Am J Gastroenterol 2011;106:508-514. doi:10.1038/ajg.2010.487

48. Vazquez-Roque MI, Camilleri M, Smyrk T, et al. A controlled trial of gluten-free diet in patients with irritable bowel syndrome-diarrhea: effects on bowel frequency and intestinal function. Gastroenterology 2013;144:903-911.e3. doi:10.1053/j.gastro.2013.01.049

49. Scarpato E, Auricchio R, Penagini F, Campanozzi A, Zuccotti GV, Troncone R. Efficacy of the gluten free diet in the management of functional gastrointestinal disorders: a systematic review on behalf of the Italian Society of Paediatrics. Ital J Pediatr 2019;45:9. doi:10.1186/ s13052-019-0606-1

50. Vernia P, Di Camillo M, Marinaro V. Lactose malabsorption, irritable bowel syndrome and self-reported milk intolerance. Dig Liver Dis 2001;33:234-239. doi:10.1016/s1590-8658(01)80713-1

51. Vernia P, Marinaro V, Argnani F, Di Camillo M, Caprilli R. Self-reported milk intolerance in irritable bowel syndrome: what should we believe? Clin Nutr 2004;23:996-1000. doi:10.1016/j.clnu.2003.12.005

52. Yang J, Deng Y, Chu H, et al. Prevalence and presentation of lactose intolerance and effects on dairy product intake in healthy subjects and patients with irritable bowel syndrome. Clin Gastroenterol Hepatol 2013;11:262-268.e1. doi:10.1016/j.cgh.2012.11.034

53. Gupta D, Ghoshal UC, Misra A, Misra A, Choudhuri G, Singh K. Lactose intolerance in patients with irritable bowel syndrome from northern India: a case-control study. J Gastroenterol Hepatol. 2007;22:2261-2265. doi:10.1111/j.1440-1746.2007.04986.x

54. Rana SV, Mandal AK, Kochhar R, Katyal R, Singh K. Lactose intolerance in different types of irritable bowel syndrome in north Indians. Trop Gastroenterol 2001;22:202-204.

55. Varjú P, Gede N, Szakács Z, et al. Lactose intolerance but not lactose maldigestion is more frequent in patients with irritable bowel syndrome than in healthy controls: A meta-analysis. Neurogastroenterol Motil 2019;31:e13527. doi:10.1111/nmo.13527

56. Böhmer CJ, Tuynman HA. The effect of a lactose-restricted diet in patients with a positive lactose tolerance test, earlier diagnosed as irritable bowel syndrome: a 5-year follow-up study. Eur J Gastroenterol Hepatol 2001;13:941-944. doi:10.1097/00042737-200108000-00011

57. Böhmer CJ, Tuynman HA. The clinical relevance of lactose malabsorption in irritable bowel syndrome. Eur J Gastroenterol Hepatol 1996;8:1013-1016. doi:10.1097/00042737-199610000-00015

58. Parker TJ, Woolner JT, Prevost AT, Tuffnell Q, Shorthouse M, Hunter JO Irritable bowel syndrome: is the search for lactose intolerance justified? Eur J Gastroenterol Hepatol 2001;13:219-225. doi:10.1097/00042737200103000-00001

59. Bozzani A, Penagini R, Velio P, et al. Lactose malabsorption and intolerance in Italians. Clinical implications. Dig Dis Sci 1986;31:13131316. doi:10.1007/BF01299809

60. Jianqin S, Leiming X, Lu X, Yelland GW, Ni J, Clarke AJ. Effects of milk containing only A2 beta casein versus milk containing both A1 and $\mathrm{A} 2$ beta casein proteins on gastrointestinal physiology, symptoms of discomfort, and cognitive behavior of people with self-reported intolerance to traditional cows' milk. Nutr J 2016;15:35. doi:10.1186/ s12937-016-0147-Z

61. Lisker R, Solomons NW, Pérez Briceño R, Ramírez Mata M. Lactase and placebo in the management of the irritable bowel syndrome: a double-blind, cross-over study. Am J Gastroenterol. 1989;84:756-762

62. Newcomer AD, Park HS, O’Brien PC, McGill DB. Response of patients with irritable bowel syndrome and lactase deficiency using unfermented acidophilus milk. Am J Clin Nutr 1983;38:257-263. doi:10.1093/ ajcn/38.2.257

63. Heghes SC, Vostinaru O, Rus LM, Mogosan C, Iuga CA, Filip L. Antispasmodic Effect of Essential Oils and Their Constituents: A Review. Molecules 2019;24:1675. doi:10.3390/molecules24091675

64. Cash BD. A Minty Breath of Fresh Air for Irritable Bowel Syndrome. Gastroenterology 2020;158:36-37.e1. doi:10.1053/j.gastro.2019.11.010

65. Chumpitazi BP, Kearns GL, Shulman RJ. Review article: the physiological effects and safety of peppermint oil and its efficacy in irritable bowel syndrome and other functional disorders. Aliment Pharmacol Ther 2018;47:738-752. doi:10.1111/apt.14519

66. Alammar N, Wang L, Saberi B, et al. The impact of peppermint oil on the irritable bowel syndrome: a meta-analysis of the pooled clinical data. BMC Complement Altern Med 2019;19:21. doi:10.1186/s12906-018-2409-0

67. Black CJ, Yuan Y, Selinger CP, et al. Efficacy of soluble fibre, antispasmodic drugs, and gut-brain neuromodulators in irritable bowel syndrome: a systematic review and network meta-analysis. Lancet Gastroenterol Hepatol 2020;5:117-131. doi:10.1016/S24681253(19)30324-3

68. Kim YS, Kim JW, Ha NY, Kim J, Ryu HS. Herbal Therapies in Functional Gastrointestinal Disorders: A Narrative Review and Clinical Implication. Front Psychiatry 2020;11:601. doi:10.3389/fpsyt.2020.00601

69. European. Medicines Agency. European Union herbal monograph on Mentha x piperita L., aetheroleum. First published: 24 July 2020. Available at: https://www.ema.europa.eu/en/documents/herbalmonograph/draft-european-union-herbal-monograph-mentha-xpiperita-1-aetheroleum-revision-1_en.pdf

70. Dimidi E, Whelan K. Food supplements and diet as treatment options in irritable bowel syndrome. Neurogastroenterol Motil 2020;32:e13951. doi:10.1111/nmo.13951

71. Cash BD, Epstein MS, Shah SM. A Novel Delivery System of Peppermint Oil Is an Effective Therapy for Irritable Bowel Syndrome Symptoms. Dig Dis Sci 2016;61:560-571. doi:10.1007/s10620-015-3858-7

72. Weerts ZZRM, Masclee AAM, Witteman BJM, et al. Efficacy and Safety of Peppermint Oil in a Randomized, Double-Blind Trial of Patients With Irritable Bowel Syndrome. Gastroenterology 2020;158:123-136. doi:10.1053/j.gastro.2019.08.026

73. Teschke R, Wolff A, Frenzel C, Eickhoff A, Schulze J. Herbal traditiona Chinese medicine and its evidence base in gastrointestinal disorders. World J Gastroenterol 2015;21:4466-4490. doi:10.3748/wjg.v21.i15.4466

74. Li CY, Ain Mohd Tahir NA, Li SC. A systematic review of integrated traditional Chinese and Western medicine for managing irritable bowel syndrome. Am J Chin Med 2015;43:385-406. doi:10.1142/ S0192415X15500251

75. Ottillinger B, Storr M, Malfertheiner P, Allescher HD. STW 5 (Iberogast $\mathrm{t}^{\oplus}$ )--a safe and effective standard in the treatment of functional gastrointestinal disorders. Wien Med Wochenschr 2013;163:65-72. doi:10.1007/s10354-012-0169-X

76. Rahimi R, Abdollahi M. Herbal medicines for the management of irritable bowel syndrome: a comprehensive review. World J Gastroenterol 2012;18:589-600. doi:10.3748/wjg.v18.i7.589 
77. Yan J, Miao ZW, Lu J, et al. Acupuncture plus Chinese Herbal Medicine for Irritable Bowel Syndrome with Diarrhea: A Systematic Review and Meta-Analysis. Evid Based Complement Alternat Med 2019;2019:7680963. doi:10.1155/2019/7680963

78. Li DY, Dai YK, Zhang YZ, et al. Systematic review and meta-analysis of traditional Chinese medicine in the treatment of constipationpredominant irritable bowel syndrome. PLoS One 2017;12:e189491. doi:10.1371/journal.pone.0189491

79. Portincasa P, Bonfrate L, Scribano ML, et al. Curcumin and Fennel Essential Oil Improve Symptoms and Quality of Life in Patients with Irritable Bowel Syndrome. J Gastrointestin Liver Dis 2016;25:151-157. doi:10.15403/jgld.2014.1121.252.ccm

80. Simrén M, Tack J. New treatments and therapeutic targets for IBS and other functional bowel disorders. Nat Rev Gastroenterol Hepatol. 2018;15(10):589-605.

81. Fan H, Zheng L, Lai Y, et al. Tongxie Formula Reduces Symptoms of Irritable Bowel Syndrome. Clin Gastroenterol Hepatol 2017;15:17241732. doi:10.1016/j.cgh.2017.06.026

82. Hill C, Guarner F, Reid G, et al. Expert consensus document. The International Scientific Association for Probiotics and Prebiotics consensus statement on the scope and appropriate use of the term probiotic. Nat Rev Gastroenterol Hepatol 2014;11:506-514. doi:10.1038/nrgastro.2014.66

83. Liu HN, Wu H, Chen YZ, Chen YJ, Shen XZ, Liu TT. Altered molecular signature of intestinal microbiota in irritable bowel syndrome patients compared with healthy controls: A systematic review and meta-analysis. Dig Liver Dis 2017;49:331-337. doi:10.1016/j.dld.2017.01.142

84. Tap J, Derrien M, Törnblom H, et al. Identification of an Intestinal Microbiota Signature Associated With Severity of Irritable Bowel Syndrome. Gastroenterology 2017;152:111-123.e8. doi:10.1053/j.gastro.2016.09.049

85. Barbara G, Cremon C, Azpiroz F. Probiotics in irritable bowel syndrome: Where are we? Neurogastroenterol Motil 2018;30:e13513. doi:10.1111/ nmo. 13513

86. Catinean A, Neag AM, Nita A, Buzea M, Buzoianu AD. Bacillus spp Spores-A Promising Treatment Option for Patients with Irritable Bowel Syndrome. Nutrients 2019;11:1968. doi:10.3390/nu11091968

87. Ford AC, Harris LA, Lacy BE, Quigley EMM, Moayyedi P. Systematic review with meta-analysis: the efficacy of prebiotics, probiotics, synbiotics and antibiotics in irritable bowel syndrome. Aliment Pharmacol Ther 2018;48:1044-1060. doi:10.1111/apt.15001

88. Niu HL, Xiao JY. The efficacy and safety of probiotics in patients with irritable bowel syndrome: Evidence based on 35 randomized controlled trials. Int J Surg 2020;75:116-127. doi:10.1016/j.ijsu.2020.01.142

89. Li B, Liang L, Deng H, Guo J, Shu H, Zhang L. Efficacy and Safety of Probiotics in Irritable Bowel Syndrome: A Systematic Review and MetaAnalysis. Front Pharmacol 2020;11:332. doi:10.3389/fphar.2020.00332

90. Su GL, Ko CW, Bercik P, et al. AGA Clinical Practice Guidelines on the Role of Probiotics in the Management of Gastrointestinal Disorders. Gastroenterology 2020;159:697-705. doi:10.1053/j.gastro.2020.05.059

91. Dale HF, Rasmussen SH, Asiller ÖÖ, Lied GA. Probiotics in Irritable Bowel Syndrome: An Up-to-Date Systematic Review. Nutrients 2019;11:2048. doi:10.3390/nu11092048

92. Doron S, Snydman DR. Risk and safety of probiotics. Clin Infect Dis 2015;60 Suppl 2:S129-S134. doi:10.1093/cid/civ085

93. Camilleri M, Ford AC. Irritable Bowel Syndrome: Pathophysiology and Current Therapeutic Approaches. Handb Exp Pharmacol 2017;239:75113. doi:10.1007/164_2016_102

94. Rawla P, Sunkara T, Raj JP. Updated review of current pharmacological and non-pharmacological management of irritable bowel syndrome. Life Sci 2018;212:176-181. doi:10.1016/j.lfs.2018.10.001
95. Johannesson E, Jakobsson Ung E, Sadik R, Ringström G. Experiences of the effects of physical activity in persons with irritable bowel syndrome (IBS): a qualitative content analysis. Scand J Gastroenterol 2018;53:1194-1200. doi:10.1080/00365521.2018.1519596

96. Johannesson E, Ringström G, Abrahamsson H, Sadik R. Intervention to increase physical activity in irritable bowel syndrome shows long-term positive effects. World J Gastroenterol 2015;21:600-608. doi:10.3748/ wjg.v21.i2.600

97. Dishman RK, Berthoud HR, Booth FW, et al. Neurobiology of exercise. Obesity (Silver Spring) 2006;14:345-356. doi:10.1038/oby.2006.46

98. Chey WD, Kurlander J, Eswaran S. Irritable bowel syndrome: a clinical review. JAMA 2015;313:949-958. doi:10.1001/jama.2015.0954

99. Mearin F, Ciriza C, Mínguez M, et al. Clinical Practice Guideline: Irritable bowel syndrome with constipation and functional constipation in the adult. Rev Esp Enferm Dig 2016;108:332-363. doi:10.17235/ reed.2016.4389/2016

100. Johannesson E, Simrén M, Strid H, Bajor A, Sadik R. Physical activity improves symptoms in irritable bowel syndrome: a randomized controlled trial. Am J Gastroenterol 2011;106:915-922. doi:10.1038/ajg.2010.480

101. Zhao Y, Xu JL, Lu SM, LG P. The effect of mountaineering exercise on somatic, psychological and mental health of the students. J China Tissue Eng Res Clin Rehabil 2007;30:6006-6009.

102. Zhou C, Zhao E, Li Y, Jia Y, Li F. Exercise therapy of patients with irritable bowel syndrome: A systematic review of randomized controlled trials. Neurogastroenterol Motil 2019;31:e13461. doi:10.1111/nmo.13461

103. Eriksson EM, Andrén KI, Kurlberg GK, Eriksson HT. Aspects of the non-pharmacological treatment of irritable bowel syndrome. World J Gastroenterol 2015;21:11439-11449. doi:10.3748/wjg.v21.i40.11439

104. Johannesson E, Jakobsson Ung E, Ringström G, Sadik R. The experiences of physical activity in irritable bowel syndrome-A qualitative study. J Clin Nurs 2019;28:3189-3199. doi:10.1111/jocn.14880

105. Zheng H, Chen R, Zhao X, et al. Comparison between the Effects of Acupuncture Relative to Other Controls on Irritable Bowel Syndrome: A Meta-Analysis. Pain Res Manag 2019;2019:2871505. doi:10.1155/2019/2871505

106. Chao GQ, Zhang S. Effectiveness of acupuncture to treat irritable bowel syndrome: a meta-analysis. World J Gastroenterol 2014;20:1871-1877. doi:10.3748/wjg.v20.17.1871

107. Wu IXY, Wong CHL, Ho RST, et al. Acupuncture and related therapies for treating irritable bowel syndrome: overview of systematic reviews and network meta-analysis. Therap Adv Gastroenterol 2019;12:1756284818820438. doi:10.1177/1756284818820438

108. Lowe C, Aiken A, Day AG, Depew W, Vanner SJ. Sham acupuncture is as efficacious as true acupuncture for the treatment of IBS: A randomized placebo controlled trial. Neurogastroenterol Motil 2017;29:e13040. doi:10.1111/nmo.13040

109. MacPherson H, Tilbrook H, Agbedjro D, Buckley H, Hewitt C, Frost C. Acupuncture for irritable bowel syndrome: 2-year follow-up of a randomised controlled trial. Acupunct Med 2017;35:17-23. doi:10.1136/ acupmed-2015-010854

110. Pei L, Geng H, Guo J, et al. Effect of Acupuncture in Patients With Irritable Bowel Syndrome: A Randomized Controlled Trial. Mayo Clin Proc 2020;95:1671-1683. doi:10.1016/j.mayocp.2020.01.042

111. Pei L, Zhu L, Sun J, Wu X, Chen L. Constipation predominant irritable bowel syndrome treated with acupuncture for regulating the mind and strengthening the spleen: a randomized controlled trial. Zhongguo Zhen Jiu 2015;35:1095-1098.

112. Rafiei R, Ataie M, Ramezani MA, et al. A new acupuncture method for management of irritable bowel syndrome: A randomized 
double blind clinical trial. J Res Med Sci 2014;19:913-917. doi:10.1155/2015/361786

113. Shi Y, Chen YH, Yin XJ, et al. Electroacupuncture versus Moxibustion for Irritable Bowel Syndrome: A Randomized, Parallel-Controlled Trial. Evid Based Complement Alternat Med 2015;2015:361786. doi:10.1155/2015/361786

114. Park SM, Kim WJ, Mun JH, et al. Adverse events associated with acupuncture: a clinicopathologic review. Int J Dermatol 2016;55:757763. doi:10.1111/ijd.12914

115. Wu J, Hu Y, Zhu Y, Yin P, Litscher G, Xu S. Systematic Review of Adverse Effects: A Further Step towards Modernization of Acupuncture in China. Evid Based Complement Alternat Med 2015;2015:432467. doi:10.1155/2015/432467

116. Black CJ, Thakur ER, Houghton LA, Quigley EMM, Moayyedi P, Ford AC. Efficacy of psychological therapies for irritable bowel syndrome: systematic review and network meta-analysis. Gut 2020;69:1441-1451. doi:10.1136/gutjnl-2020-321191

117. Stanculete MF, Matu S, Pojoga C, Dumitrascu DL. Coping strategies and irrational beliefs as mediators of the health-related quality of life impairments in irritable bowel syndrome. J Gastrointestin Liver Dis 2015;24:159-164. doi:10.15403/jgld.2014.1121.242.strt

118. Radu M, Moldovan R, Pintea S, Băban A, Dumitrascu D. Predictors of outcome in cognitive and behavioural interventions for irritable bowel syndrome. A meta-analysis. J Gastrointestin Liver Dis 2018;27:257-263. doi:10.15403/jgld.2014.1121.273.bab

119. Payne A, Blanchard EB. A controlled comparison of cognitive therapy and self-help support groups in the treatment of irritable bowel syndrome. J Consult Clin Psychol 1995;63:779-786. doi:10.1037//0022006x.63.5.779

120. Ljótsson B, Falk L, Vesterlund AW, et al. Internet-delivered exposure and mindfulness based therapy for irritable bowel syndrome--a randomized controlled trial. Behav Res Ther 2010;48:531-539. doi:10.1016/j. brat.2010.03.003

121. Ljótsson B, Hedman E, Lindfors $\mathrm{P}$, et al. Long-term follow-up of internet-delivered exposure and mindfulness based treatment for irritable bowel syndrome. Behav Res Ther 2011;49:58-61. doi:10.1016/j. brat.2010.10.006

122. Lackner JM, Jaccard J, Keefer L, et al. Improvement in Gastrointestinal Symptoms After Cognitive Behavior Therapy for Refractory Irritable Bowel Syndrome. Gastroenterology 2018;155:47-57. doi:10.1053/j. gastro.2018.03.063

123. Lindfors $P$, Unge P, Arvidsson P, et al. Effects of gut-directed hypnotherapy on IBS in different clinical settings-results from two randomized, controlled trials. Am J Gastroenterol 2012;107:276-285. doi:10.1038/ajg.2011.340

124. Moser G, Trägner S, Gajowniczek EE, et al. Long-term success of GUT-directed group hypnosis for patients with refractory irritable bowel syndrome: a randomized controlled trial. Am J Gastroenterol 2013;108:602-609. doi:10.1038/ajg.2013.19

125. Flik CE, Laan W, Zuithoff NPA, et al. Efficacy of individual and group hypnotherapy in irritable bowel syndrome (IMAGINE): a multicentre randomised controlled trial. Lancet Gastroenterol Hepatol 2019;4:20-31. doi:10.1016/S2468-1253(18)30310-8

126. Menees S, Chey W. The gut microbiome and irritable bowel syndrome. F1000Res 2018;7(F1000 Faculty Rev):1029. doi:10.12688/ f1000research.14592.1

127. Carroll IM, Ringel-Kulka T, Siddle JP, Ringel Y. Alterations in composition and diversity of the intestinal microbiota in patients with diarrhea-predominant irritable bowel syndrome. Neurogastroenterol Motil 2012;24:521-530. doi:10.1111/j.13652982.2012.01891.x

128. Bhattarai Y, Muniz Pedrogo DA, Kashyap PC. Irritable bowel syndrome: a gut microbiota-related disorder? Am J Physiol Gastrointest Liver Physiol 2017;312:G52-G62. doi:10.1152/ajpgi.00338.2016

129. El-Salhy M, Hatlebakk JG, Gilja OH, Bråthen Kristoffersen A, Hausken T. Efficacy of faecal microbiota transplantation for patients with irritable bowel syndrome in a randomised, double-blind, placebo-controlled study. Gut 2020;69:859-867. doi:10.1136/gutjnl-2019-319630

130. Holvoet T, Joossens M, Vázquez-Castellanos JF, et al. Fecal Microbiota Transplantation Reduces Symptoms in Some Patients With Irritable Bowel Syndrome With Predominant Abdominal Bloating: Shortand Long-term Results From a Placebo-Controlled Randomized Trial. Gastroenterology 2021;160:145-157.e8. doi:10.1053/j. gastro.2020.07.013

131. Aroniadis OC, Brandt LJ, Oneto C, et al. Faecal microbiota transplantation for diarrhoea-predominant irritable bowel syndrome: a double-blind, randomised, placebo-controlled trial. Lancet Gastroenterol Hepatol 2019;4:675-685. doi:10.1016/S2468-1253(19)30198-0

132. Halkjær SI, Christensen AH, Lo BZS, et al. Faecal microbiota transplantation alters gut microbiota in patients with irritable bowel syndrome: results from a randomised, double-blind placebo-controlled study. Gut 2018;67:2107-2115. doi:10.1136/gutjnl-2018-316434

133. Johnsen PH, Hilpüsch F, Cavanagh JP, et al. Faecal microbiota transplantation versus placebo for moderate-to-severe irritable bowel syndrome: a double-blind, randomised, placebo-controlled, parallelgroup, single-centre trial. Lancet Gastroenterol Hepatol 2018;3:17-24 doi:10.1016/S2468-1253(17)30338-2

134. Holster S, Lindqvist CM, Repsilber D, et al. The Effect of Allogenic Versus Autologous Fecal Microbiota Transfer on Symptoms, Visceral Perception and Fecal and Mucosal Microbiota in Irritable Bowel Syndrome: A Randomized Controlled Study. Clin Transl Gastroenterol 2019;10:e00034. doi:10.14309/ctg.0000000000000034

135. Lahtinen P, Jalanka J, Hartikainen A, et al. Randomised clinical trial: faecal microbiota transplantation versus autologous placebo administered via colonoscopy in irritable bowel syndrome. Aliment Pharmacol Ther 2020;51:1321-1331. doi:10.1111/apt.15740

136. Flik CE, Bakker L, Laan W, van Rood YR, Smout AJ, de Wit NJ. Systematic review: The placebo effect of psychological interventions in the treatment of irritable bowel syndrome. World J Gastroenterol 2017;23:2223-2233. doi:10.3748/wjg.v23.i12.2223

137. Myneedu K, Deoker A, Schmulson MJ, Bashashati M. Fecal microbiota transplantation in irritable bowel syndrome: A systematic review and meta-analysis. United European Gastroenterol J 2019;7:1033-1041. doi:10.1177/2050640619866990

138. Xu D, Chen VL, Steiner CA, et al. Efficacy of Fecal Microbiota Transplantation in Irritable Bowel Syndrome: A Systematic Review and Meta-Analysis. Am J Gastroenterol 2019;114:1043-1050. doi:10.14309/ ajg.0000000000000198

139. Sperber AD, Bangdiwala SI, Drossman DA, et al. Worldwide Prevalence and Burden of Functional Gastrointestinal Disorders, Results of Rome Foundation Global Study. Gastroenterology 2021;160:99-114.e3. doi:10.1053/j.gastro.2020.04.014

140. Zhou S, Liu X, Wang X, et al. Pharmacological and non-pharmacological treatments for irritable bowel syndrome: Protocol for a systematic review and network meta-analysis. Medicine (Baltimore) 2019;98:e16446. doi:10.1097/MD.0000000000016446 